\title{
A Comparison of Genetic Diversity of COX-III Gene in Lowland Chickens and Tibetan Chickens
}

\author{
Xueqin Liu, Pu Zhang, Gongying Zhang, Sichen Li, Long Zhang, Zhongxian Xu, \\ Tianyuan Ma, and Diyan Li \\ Institute of Animal Genetics and Breeding, College of Animal Science and Technology, Sichuan Agricultural University, \\ Chengdu, China \\ Correspondence should be addressed to Diyan Li; diyanli@sicau.edu.cn
}

Received 21 January 2017; Revised 3 May 2017; Accepted 25 May 2017; Published 3 July 2017

Academic Editor: Daniele Corsaro

Copyright (C) 2017 Xueqin Liu et al. This is an open access article distributed under the Creative Commons Attribution License, which permits unrestricted use, distribution, and reproduction in any medium, provided the original work is properly cited.

\begin{abstract}
To obtain a full understanding of the genetic diversity of the cytochrome oxidase III gene (COX-III) and its association with high altitude adaptation in Tibetan chickens, we sequenced COX-III in 12 chicken populations (155 Tibetan chickens and 145 other domestic chickens). We identified a total of 11 single nucleotide polymorphisms (SNPs) and 12 haplotypes (Hal-Ha12). Low genetic diversity (haplotype diversity $=0.531 \pm 0.087$, nucleotide diversity $=0.00125$ ) was detected for COX-III, and haplotype diversity of Tibetan chicken populations $(0.750 \pm 0.018)$ was markedly higher than lowland chicken populations $(0.570 \pm 0.028)$. Obvious genetic differentiation (nucleotide divergence $=0.092 \sim 0.339$ ) and conspicuous gene communication (gene flow $=0.33 \sim 32.22$ ) among 12 populations suggested that Tianfu black-bone fowl (white feather) was possibly introduced from Tibetan chicken. SNP m.10587 T > C affects the specific functions of the COX enzyme. Haplotype Ha3 was found in Tibetan chickens, and SNP m.10115G $>$ A caused an amino acid substitution (Val62Ile) associated with phospholipid binding, while mutations m.10017C > A and m.10555G $>$ A and the previously reported SNP m.10065T $>$ C reduced the hydropathy index to some extent. Together, this indicates that the mitochondrial membrane is more hydrophobic in Tibetan chickens.
\end{abstract}

\section{Introduction}

Domestic chickens fulfill various roles ranging from food and entertainment to religion and ornamentation [1]. Tibetan chicken is a widely distributed aboriginal chicken breed found at altitudes ranging from 2200 to $4100 \mathrm{~m}$; it has adapted well to high altitudes after over the years of living on the plateau [2]. Generally, long-term exposure to hypoxia in animals reduces metabolic activity, retards development, and increases embryo mortality [3]. However, Tibetan chicken has developed an adaptive mechanism to hypoxia, demonstrated by its increased hatchability and survival rate compared with lowland chicken breeds in high altitude areas of Tibet. With its low weight, small size, and strong chest and legs, the appearance and behaviour of the Tibetan chicken resemble those of the Cochin-Chinese red jungle fowl (Gallus gallus gallus), making it particularly good at flying and foraging on the plateau alpine region [2-5]. The breed is also important to the resources used to expand the industry in cold areas of high altitude in China.

Avian species living at high altitudes are characterized by the high oxygen affinity of their haemoglobin $[6,7]$. Tibetan birds can improve their physiological performance by enhancing their oxygen transport capacity, which has yielded important insights into the genetic basis of adaptation involving haemoglobin as an oxygen carrier $[8,9]$. Taking into account the importance of using oxygen more efficiently under hypoxic conditions, information mining for the cellular respiratory chain is a valuable way of understanding the hypoxia response and adaptation mechanisms.

Mitochondrial DNA (mtDNA) sequences are widely used in molecular evolutionary studies. These sequences are useful for estimating times of species and population divergences, comparisons of relative rates of evolution, and phylogenetic inferences within and between vertebrate species [10]. The complete sequence of the chicken mtDNA is 16,775 base pairs 
and contains 13 protein coding genes, two rRNA genes, and 22 tRNA genes [11]. Cytochrome c oxidase (COX), the terminal enzyme of the mitochondrial respiratory chain, contains 14 protein subunits in mammals, of which three (COX-I, COXII, and COX-III) are synthesized in the mitochondria [12]. Mitochondrial cytochrome c oxidase subunit I (COX-I) is the central catalytic subunit of cytochrome c oxidase (complex IV), and COX-I gene is used as a standard marker for DNA bar coding to enable species identification in animals [3, 13]. The COX-III protein is an important element in regulating the efficiency of proton translocation in cytochrome oxidase over several turnovers [14]. The COX-III gene of bar-headed geese contained a nonsynonymous substitution (Trp-116 $\rightarrow$ Arg) that resulted in a major functional change of amino acid class. This mutation was predicted by structural modeling to alter the interaction between COX-III and COXI, which contributed to adaptation in mitochondrial enzyme kinetics and $\mathrm{O} 2$ transport capacity and may finally contribute to the exceptional ability of bar-headed geese to fly at extreme heights [15].

COX has been investigated in several biological studies $[3,6,16,17]$, but studies of COX-III have rarely been reported in chickens. However, COX-III is an important component of the respiratory chain and is very conserved among species. Adaptive changes in COX activity can alter the ATP supply derived from oxidative phosphorylation during hypoxia [9]. The quaternary structure formed by different protein subunits is stabilized mainly through hydrophobic interactions in spite of hydrogen bonding and the van der Waals force is also important. We hypothesized that the stability of the COX holoenzyme three-dimensional structure would increase in line with increases in COX-III protein hydrophobicity. Therefore, in the present study, based on the assumption that cytochrome $\mathrm{c}$ oxidase activity is more stable in Tibetan chickens, we analyzed COX-III SNPs in 12 chicken populations (five lowland and seven highland populations) to better understand the COX-III genetic diversity and to determine the contribution of specific SNPs to high altitude adaptations in Tibetan chickens.

\section{Materials and Methods}

In all experimental populations, 5 populations (Muchuan, Emei, Jiuyuan, Black-Tianfu, and White-Tianfu) belonged to lowland chickens; the other 7 populations (Haiyan, Doilungdêqên, Ganzi, Nyingchi, Diqing, Shannan, and Shigatse) which belonged to Tibetan chickens were collected (Table 1). Blood samples were collected from the wing vein. No bird was slaughtered or unexpectedly injured during sampling. The protocol was approved by the Committee on the Care and Use of Laboratory Animals of the StateLevel Animal Experimental Teaching Demonstration Center of Sichuan Agricultural University (Approval ID: Decree number S20160906).

2.1. DNA Extraction, Amplification, and Sequencing. We extracted mtDNA by salt extraction method [18]. PCR used the known primer pairs F9797: 5' -ACCAATAATACCATCAATCTCC- $3^{\prime}$ and R10830: $5^{\prime}$ CGCTTAGTAGAAAGGATAGTGAG-3 ${ }^{\prime}[19,20]$. PCR amplification was performed in a $50 \mu \mathrm{l}$ volume with $100-150 \mathrm{ng}$ of genomic DNA, $25 \mathrm{mM}$ $\mathrm{MgCl} 2,2.5 \mathrm{mM}$ of dNTP mixture, $2 \mathrm{mM}$ each primer, $5 \mu \mathrm{l}$ of 10x buffer, and 1.25 U LA Taq polymerase (Takara, Dalian, China) under the following conditions: denaturation at $94^{\circ} \mathrm{C}$ for $5 \mathrm{~min}$, then 35 cycles of $94^{\circ} \mathrm{C}$ for $30 \mathrm{~s}, 55^{\circ} \mathrm{C}$ for $30 \mathrm{~s}$, and $72^{\circ} \mathrm{C}$ for $60 \mathrm{~s}$, followed by a final extension at $72^{\circ} \mathrm{C}$ for 7 min [19]. PCR products were verified on $1.5 \%$ agarose gels, and specific bands were purified using the TIANgel Midi Purification Kit (Tiangen Biotech, Beijing, China). Purified PCR products were sequenced in both directions using the Big Dye Terminator v. 3.1 Cycle Sequencing Kit (Applied Biosystems, Foster City, CA) on the ABI Prism 3100 DNA sequencer (Applied Biosystems) according to the manufacturer's instructions.

2.2. Sequence Data Analysis and Statistical Analysis. Raw sequences were aligned and edited by DNAstar software (DNAstar Inc. Madison, WI, USA). We exported all sequences as an aligned FASTA file. Sequence variations were identified using MEGA 6.0 software [21]. Standard population genetics statistics, including haplotypes and number of haplotypes, haplotype diversity within each group $(H d)$, nucleotide diversity $(P i)$, nucleotide divergence $(D x y)$, net genetic distance $(D a)$, coefficient of differentiation (Gst), and gene flow $(\mathrm{Nm})$, Tajima's $D$ value neutral test, were defined using DnaSP V5 software [22], whereas median joining network analysis was performed using program network 4.611 (http://www.fluxus-engineering.com/sharenet .htm). The complete mitochondrial genome sequence of the red jungle fowl was used as the reference sequence (GenBank accession number: NC_001323).

Analysis of molecular variance (AMOVA) was estimated using Arlequin 3.0 software [23]. Bayesian inference was performed as previously described [24]. The optimal model for each data set was estimated by the program Modeltest 3.7 [25]. The program BEAUti v1.5.3 (distributed with BEAST) was used to create the input file to run in BEAST (http://beast.bio.ed.ac.uk/). Samples from the posterior were summarized on the maximum clade credibility tree using the program TreeAnnotator v1.4.8 (distributed with BEAST) and visualized using the program FigTree v1.3.1 (http://tree.bio.ed.ac.uk/software/figtree/). Statistical differences in COX-III haplotype frequencies between Tibetan chickens and lowland chickens were analyzed using Fisher's exact test; odds ratios (OR) and $95 \%$ confidence intervals ( $95 \%$ CIs) were also calculated. $P$ value $<0.05$ was taken into account as statistical significance. The bioinformatics platform MitoTool (http://www.mitotool.org/) was used to analyze haplotype distribution frequencies between Tibetan chickens and lowland chickens [26]. $P<0.05$ were taken to be statistically significant.

Protter [27] was used to annotate and predict protein sequence features of the COX-III protein (http://wlab.ethz.ch/ protter/start/). Hydropathy plot of the COX-III protein was predicted using the Tmpred Program [28] (http://www.ch .embnet.org/software/TMPRED_form.html). 


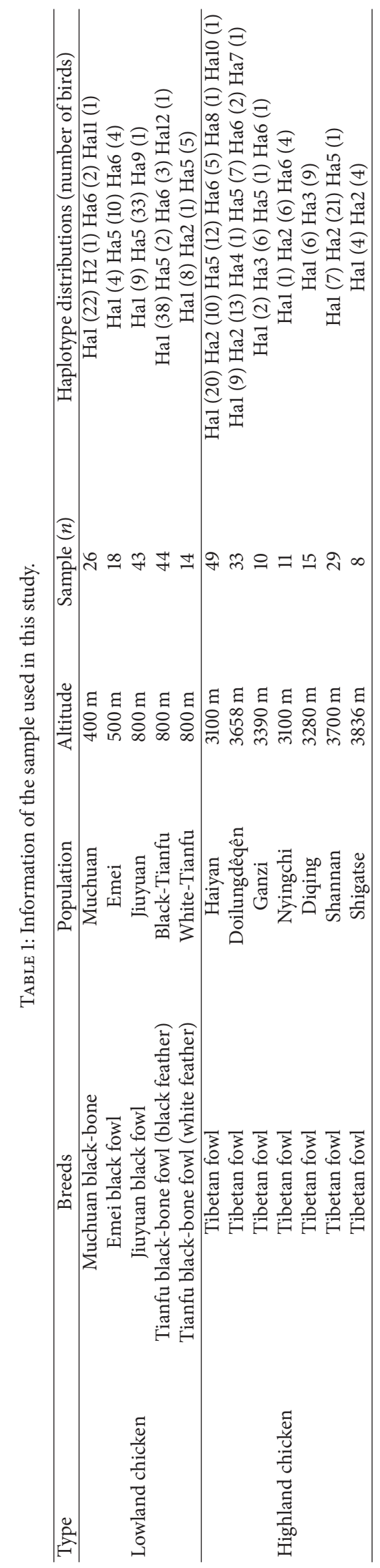




\section{Results}

3.1. Nucleotide Diversity of COX-III. The length of 300 COXIII sequences was truncated into $784 \mathrm{bp}$ (GenBank accession numbers: NC_001323; no insertion/deletions were detected). We calculated the overall base composition of COX-III from the 12 chicken populations using MEGA 6.0. Cytosine (C) was shown to be the rarest nucleotide $(16 \%)$ with guanine $(\mathrm{G})$ to the most common (32\%). The A $+\mathrm{T} \%$ was around half of COX-III (52\%).

A total of 11 SNPs (including six singleton sites and five parsimony-informative sites (with no insertions/deletions)) accounted for $1.403 \%$ of the total $784 \mathrm{bp}$ COX-III sequence from 300 individuals. Table 2 summarized the number of haplotypes, $\mathrm{Hd}, \mathrm{Pi}$, and other information within each group. The number of variable sites in each population varies from 1 in Diqing and Shigatse to 7 in Haiyan, and the sequences from Doilungdêqên, Ganzi, Nyingchi, Shannan, were 5, 5, 3, and 2, respectively. The highest haplotype diversity was found in Haiyan and Doilungdêqên. While the average number of nucleotide differences in Emei from lowland chickens was greater than others. Taken together, a total of 8 haplotypes were identified in lowland chickens, and the overall haplotype diversity, nucleotide diversity, and average nucleotide differences were $0.570 \pm 0.028,0.00155$, and 1.216 , respectively. In Tibetan chickens, a total of 9 haplotypes were identified, and the overall haplotype diversity, nucleotide diversity, and average nucleotide differences were $0.750 \pm 0.018,0.0016$, and 1.250 , respectively. The result showed that the genetic diversity of the Tibetan chicken was noticeably higher than that of lowland chickens. After Tajima's $D$ value neutral test, all $P$ values were greater than 0.1 ; therefore, the 12 populations belong to neutral mutations.

3.2. Nucleotide Divergence and Net Genetic Distance among Populations. Nucleotide polymorphism among populations can be represented by nucleotide divergence (Dxy) and net genetic distance in nucleotides $(\mathrm{Da})$. Within the 12 chicken populations, the average $D x y$ was $0.062 \%$ (range $0.092 \%-0.339 \%$ ) and the average $D a$ was $0.196 \%$ (range $0 \%-0.216 \%$ ) (Table 3$)$. The largest $D a(0.216 \%)$ was found between Diqing and Jiuyuan black chickens (between Tibetan chickens and lowland chickens), and the smallest $\mathrm{Da}(0 \%)$ was observed between Haiyan and Doilungdêqên chickens; the smallest Dxy (0.092\%) was observed between BlackTianfu and Muchuan black chickens (within lowland chickens) and the largest $D x y(0.339 \%)$ was found between Diqing and Emei chickens (between Tibetan chickens and lowland chickens).

To pinpoint the most and least closely related populations, we used net genetic distance in nucleotides ( $D a$ ) to demonstrate that Haiyan and Doilungdêqên are most similar in highland chickens $(D a=0 \%, D x y=0.177 \%)$ and that Muchuan black-bone fowl and Tianfu black-bone fowl (black feather) are most similar in lowland chickens $(D a=0.002 \%, D x y=0.092 \%)$. Conversely, Tibetan chickens (Diqing) appear more distantly related to Jiuyuan black fowl $(D a=0.216 \%, D x y=0.308 \%)$ and Emei black fowl $(D a=0.187 \%, D x y=0.339 \%)$.
These findings are conclusive of definite genetic differentiation between different groups of chickens, with the strongest differentiation seen between Tibetan chickens and lowland chickens. Net genetic distances in nucleotides $(\mathrm{Da})$ are substantially consistent with the outcome of nucleotide differences (Dxy).

3.3. Coefficient of Differentiation (Gst) and Gene Flow (Nm) between Populations. The coefficient of differentiation (Gst) can reveal the extent of gene flow and genetic drift to some extent, while gene flow can uncover possible gene infiltration among populations. In lowland and Tibetan chicken populations, distinct gene exchange (Gst $=0.01, \mathrm{Nm}=25.21$; $G s t=0.05, N m=4.9)$ was detected between White-Tianfu (lowland chickens) and Haiyan and Doilungdêqên (Tibetan chickens). However, at the same altitude, obvious genetic differentiation was found in lowland geographical populations $(P<0.05)$ while conspicuous gene communication (Gst $=0.01 \mathrm{Nm}=29.34$ ) was only detected in Muchuan and Black-Tianfu chickens. Obvious genetic differentiation appears to have occurred in Tibetan chickens (Table 4). This indicates that differences in varieties caused genetic differentiation and that the introduction of varieties of different regions led to gene exchange.

3.4. Analysis of Molecular Variance. AMOVA showed that the percentage of variation within populations $(70.43 \%)$ was greater than that between populations (29.57\%). Fst value was $0.2957\left({ }^{* *} P<0.01\right)$ which implied that the genetic divergence within populations was significant. The results indicate that the twelve geographic populations do not produce largely genetic differentiation, while the genetic diversity in COX-III gene mainly comes from within the populations (Table 5).

3.5. Sequence Variations in COX-III Gene. We detected seven synonymous mutations (m.10081A $>\mathrm{G}, \mathrm{m} .10162 \mathrm{G}>\mathrm{A}$, m.10270G $>$ A, m.10336A $>$ G, m.10369G $>A$, m.10587 T $>$ C, and $\mathrm{m} .10809 \mathrm{C}>\mathrm{T}$ ) and four nonsynonymous substitutions (m.10017C > A, m.10112 G>A, m.10115G > A, and m.10555G > A) in COX-III. Table 6 shows the observed allele frequencies in each polymorphic site between Tibetan chicken and lowland chicken breeds. After using Pearson chi-square test, we found that three SNPs compared with lowland chickens in COXIII gene (m.10115G $>$ A, m.10270G $>A$, and $10587 \mathrm{~T}>\mathrm{C}$ ) were significantly different with the allele frequency of $9.7 \%$, $91.0 \%$, and $86.5 \%\left({ }^{*} P<0.05\right)$ in Tibetan chicken, respectively. Similarly, in lowland chicken, the SNP m.10081A $>$ G was significantly different from Tibetan chicken with the allele frequency of $57.2 \%$. The four SNPs (m.10017C $>$ A and m.10369G $>$ A, m.10555G $>A$, and m.10809C $>$ T) were only distributed in Tibetan chicken breeds with the allele frequency of only $0.6 \%$ and $7.7 \%$, while the other three SNPs (m.10112 G>A, m.10162G $>A$, and m.10336A $>$ G) were only distributed in lowland chicken with the allele frequency of $0.7 \%$. We consider that the seven SNPs showed nonsignificant difference between Tibetan chicken and lowland chicken breeds $(P>0.05)$. 


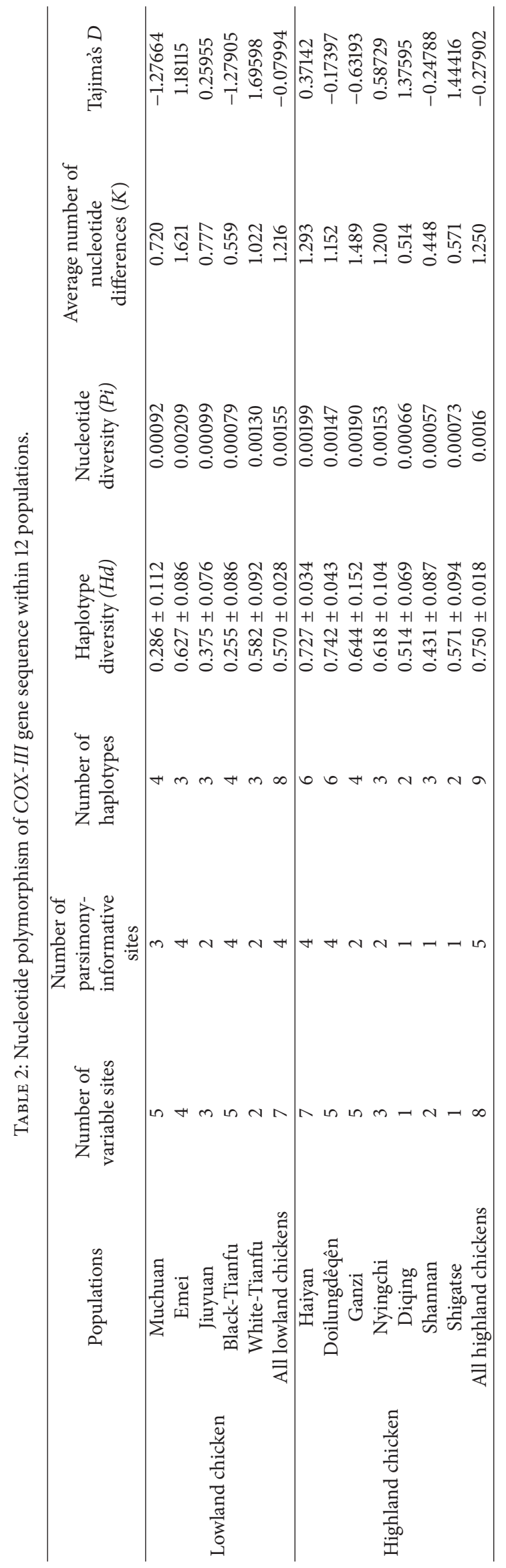




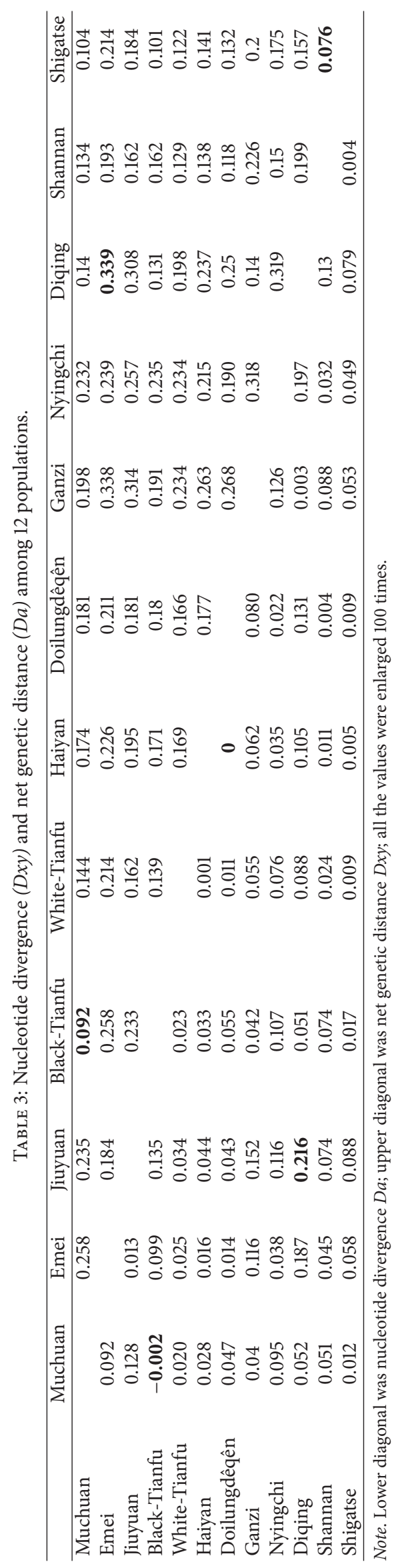




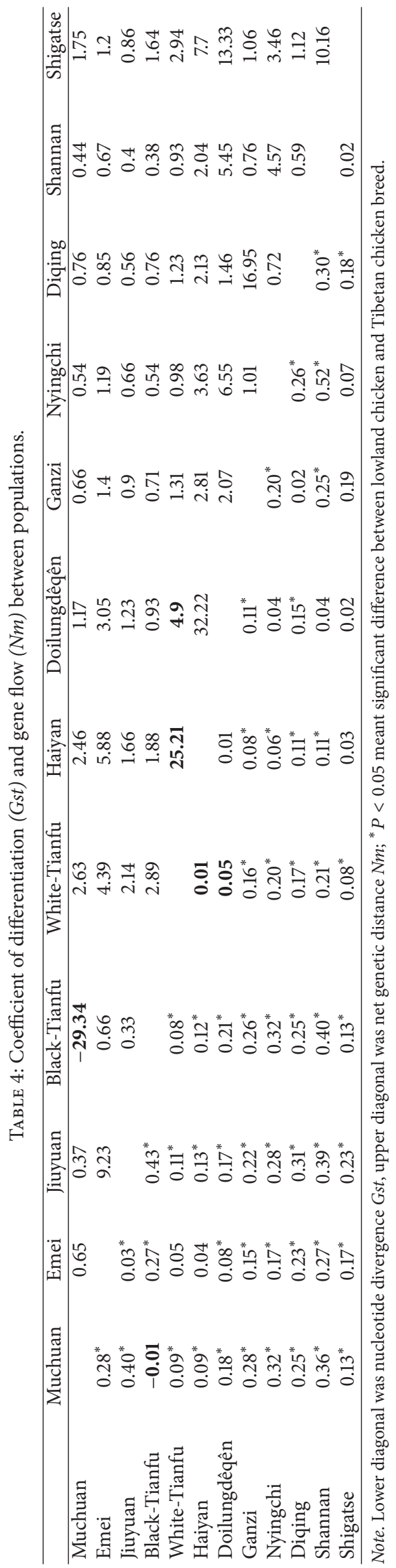


TABLE 5: Analysis of molecular variance of COX-III sequences in 12 populations.

\begin{tabular}{lccccc}
\hline Source of variation & Sum of squares & $\mathrm{df}$ & Variance components & Percentage & Fixation index \\
\hline Among populations & 56.807 & 11 & $0.19(\mathrm{Va})$ & 29.57 & 70.43 \\
Within populations & 132.731 & 288 & $0.46(\mathrm{Vb})$ & $0.2957^{* *}$ \\
\hline
\end{tabular}

Note. Fixation index: $0.2957 ;{ }^{* *} P<0.01$.

TABLE 6: Distribution of SNPs in COX-III gene in Tibetan chickens and lowland chickens.

\begin{tabular}{|c|c|c|c|c|}
\hline \multirow{2}{*}{ SNP sites } & \multicolumn{3}{|c|}{ Allele distribution } & \multirow{2}{*}{$P$ value in Pearson chi-square test } \\
\hline & Allele & $\mathrm{TC}^{\mathrm{a}}$ & $\mathrm{LC}^{\mathrm{b}}$ & \\
\hline \multirow{2}{*}{10017} & $\mathrm{C}$ & $154(99.4 \%)$ & $145(100 \%)$ & \multirow{2}{*}{0.33263} \\
\hline & A & $1(0.6 \%)$ & $0(0 \%)$ & \\
\hline \multirow{2}{*}{10081} & $\mathrm{~A}$ & $90(58.1 \%)$ & $62(42.8 \%)$ & \multirow{2}{*}{$0.00805^{*}$} \\
\hline & G & $65(41.9 \%)$ & $83(57.2 \%)$ & \\
\hline \multirow{2}{*}{10112} & G & $155(100 \%)$ & $144(99.3 \%)$ & \multirow{2}{*}{0.30037} \\
\hline & $\mathrm{A}$ & $0(0 \%)$ & $1(0.7 \%)$ & \\
\hline \multirow{2}{*}{10115} & G & $140(90.3 \%)$ & $145(100 \%)$ & \multirow{2}{*}{$0.00012^{*}$} \\
\hline & $\mathrm{A}$ & $15(9.7 \%)$ & $0(0 \%)$ & \\
\hline \multirow{2}{*}{10162} & G & $155(100 \%)$ & $144(99.3 \%)$ & \multirow{2}{*}{0.30037} \\
\hline & A & $0(0 \%)$ & $1(0.7 \%)$ & \\
\hline \multirow{2}{*}{10270} & G & $14(9 \%)$ & $136(93.8 \%)$ & \multirow{2}{*}{$9.62 \times 10^{-49^{*}}$} \\
\hline & $\mathrm{A}$ & $141(91.0 \%)$ & $9(6.2 \%)$ & \\
\hline \multirow{2}{*}{10336} & A & $155(100 \%)$ & $144(99.3 \%)$ & \multirow{2}{*}{0.30037} \\
\hline & G & $0(0 \%)$ & $1(0.7 \%)$ & \\
\hline \multirow{2}{*}{10369} & G & $154(99.4 \%)$ & $145(100 \%)$ & \multirow{2}{*}{0.33263} \\
\hline & A & $1(0.6 \%)$ & $0(0 \%)$ & \\
\hline \multirow{2}{*}{10555} & G & $154(99.4 \%)$ & $145(100 \%)$ & \multirow{2}{*}{0.33263} \\
\hline & A & $1(0.6 \%)$ & $0(0 \%)$ & \\
\hline \multirow{2}{*}{10587} & $\mathrm{~T}$ & $21(13.5 \%)$ & $94(64.8 \%)$ & \multirow{2}{*}{$6.92 \times 10^{-20 *}$} \\
\hline & $\mathrm{C}$ & $134(86.5 \%)$ & $51(35.2 \%)$ & \\
\hline \multirow{2}{*}{10809} & $\mathrm{C}$ & $143(92.3 \%)$ & $136(93.8 \%)$ & \multirow{2}{*}{0.60255} \\
\hline & $\mathrm{T}$ & $12(7.7 \%)$ & $9(6.2 \%)$ & \\
\hline
\end{tabular}

Note: relative content in parentheses meant the number of birds in corresponding allele in the specific polymorphic site; ${ }^{*} P<0.05$ meant significant difference between lowland chicken and Tibetan chicken breeds; ${ }^{\mathrm{a}} \mathrm{TC}$ was the abbreviations for Tibetan chicken; ${ }^{\mathrm{b}} \mathrm{LC}$ was the abbreviations for lowland chicken.

3.6. Median Joining Network of Haplotypes and Phylogenetic Analysis. We identified 12 haplotypes (Hal-Ha12) in 300 chickens from the 12 different populations (Table 7). The median joining network was constructed using the 12 haplotypes. Three clusters (A, B, and C) were clearly defined from the network with substantial mutation distances visible between the clusters (Figure 1). Hal, Ha3, Ha8, Ha9, and $\mathrm{Ha} 2$ were restricted to cluster A: Hal was the dominant haplotype, present in $87.8 \%$ of all individuals in cluster A (130/148 = 87.8\%); Ha2, Ha5, Ha10, and Ha11 were restricted to cluster B: Ha5 was the dominant haplotype, present in $55.03 \%$ of all individuals in cluster B $(71 / 129=55.03 \%)$; Ha4, Ha6, and $\mathrm{Ha} 7$ were restricted to cluster $\mathrm{C}$ : $\mathrm{Ha} 6$ was the dominant haplotype, present in $91.3 \%$ of all individuals in cluster B $(21 / 23=99.3 \%)$. The ancestral haplotype was mainly distributed in the center of the median joining network, with derivative haplotypes spreading outwards from it. This indicates that $\mathrm{Ha} 1, \mathrm{Ha} 2$, and $\mathrm{Ha} 4$ are the earliest haplotypes.

We also used the Meleagris gallopavo as an outside group to construct a phylogenetic tree using the Bayesian method.
The correlation of the 12 haplotypes is shown in Figure 2, and three clusters can also be seen in the Bayesian tree.

3.7. Association between Haplotype Distribution and Altitude Adaptation. Haplotypes of sample sizes under five were not taken into account here. After reviewing the level of significance by Bonferroni correction, haplotype $\mathrm{Ha} 2$ was found to be significantly associated with high altitude adaptation at the 0.05 level $\left(P\right.$ value, $1.911 \times 10^{-14^{*}}$; OR, 30.375, 95\% CI, 7.332-125.837). Haplotypes $\mathrm{Ha} 1$ and $\mathrm{Ha} 5$ also appear to be significantly associated with lowland adaptation ( $\mathrm{H1}: P$ value, 0.001; OR, 1.918; 95\% CI, 1.286-2.860; H5: $P$ value, 0.00022, OR, 2.685, CI, 1.567-4.598), whereas haplotype Ha6 does not seem significantly associated with altitude adaptation at the 0.05 level ( $P$ value, 0.658 ; OR, $0.74736,95 \%$ CI, $0.310-1.801$ ) (Table 8).

3.8. Prediction and Analysis of Secondary Structure Changes in the COX-III Protein. Protein sequencing showed that the Tibetan chicken-specific nonsynonymous COX-III variants 


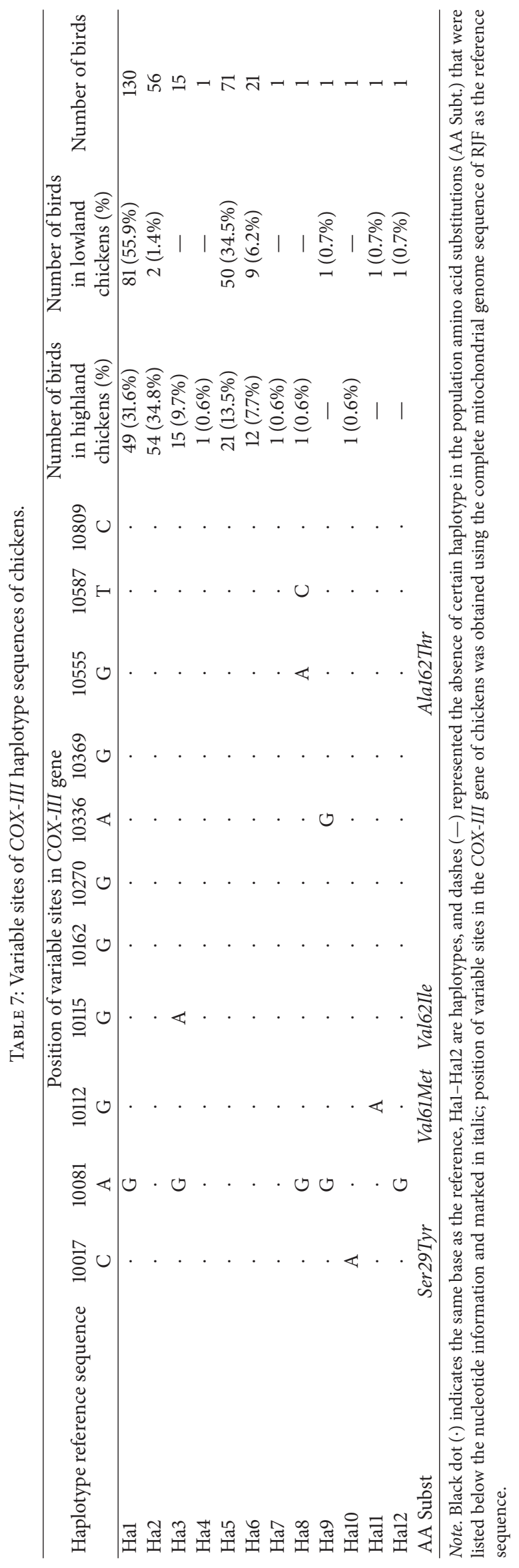


TABLE 8: Patterns of haplotype distribution in COX-III gene.

\begin{tabular}{|c|c|c|c|c|c|}
\hline Haplotype & Number of Tibetan chickens & Number of lowland chickens & $P$ value & OR & $95 \%$ CIs \\
\hline Hal & 49 & 81 & $0.00035^{*}$ & 0.484 & $0.32515-0.72169$ \\
\hline $\mathrm{Ha} 2$ & 54 & 2 & $1.911 \times 10^{-14^{*}}$ & 30.375 & $7.332-125.837$ \\
\hline Ha3 & 15 & 0 & $0.00006^{*}$ & - & - \\
\hline $\mathrm{Ha} 4$ & 1 & 0 & 1.000 & - & - \\
\hline Ha5 & 21 & 50 & $0.00008^{*}$ & 0.349 & $0.20373-0.59712$ \\
\hline Ha6 & 12 & 9 & 0.66178 & 1.257 & $0.52175-3.030$ \\
\hline Ha7 & 1 & 0 & 1.000 & - & - \\
\hline $\mathrm{Ha} 8$ & 1 & 0 & 1.000 & - & - \\
\hline Ha9 & 0 & 1 & 0.483 & - & - \\
\hline Ha10 & 1 & 0 & 1.000 & - & - \\
\hline Hall & 0 & 1 & 0.483 & - & - \\
\hline Ha12 & 0 & 1 & 0.483 & - & - \\
\hline Total & 155 & 145 & & & \\
\hline
\end{tabular}

Note: OR: odds ratio. OR $<1$ mean haplotype may be negatively associated with high-altitude adaptation; OR = 1 mean haplotype is not associated with highaltitude adaptation; OR $>1$, haplotype may be surely associated with high-altitude adaptation. CIs: confidence intervals; ${ }^{*} P<0.05$ meant significant difference between lowland chicken and Tibetan chicken breeds.

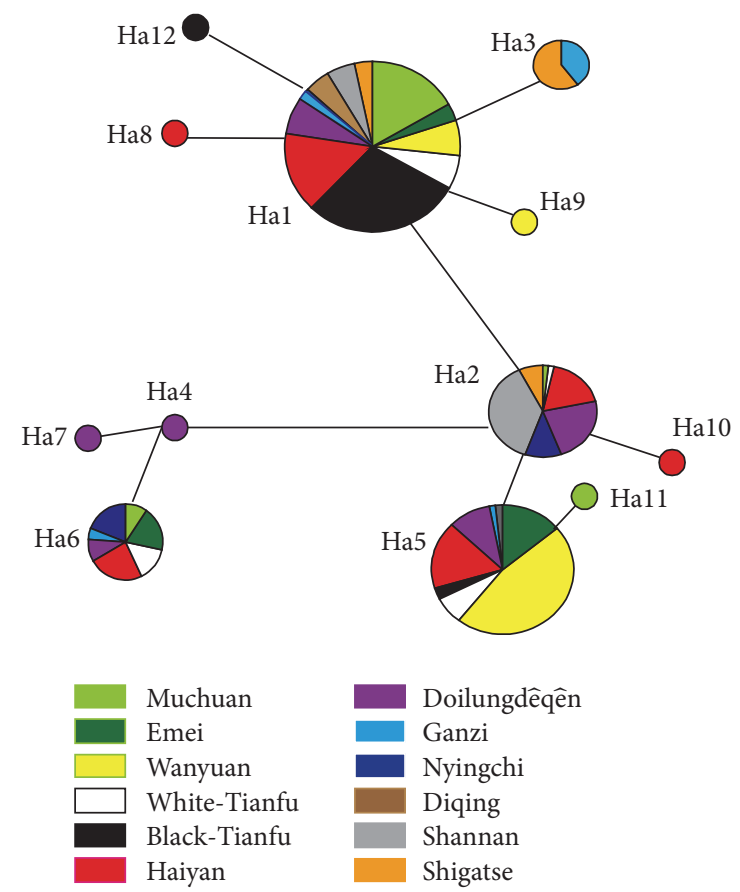

FIGURE 1: Median joining network of COX-III gene haplotypes. Geographic of samples as showed by different colors. Note: population of different regions is replaced by different colors.

m.10017C $>$ A and m.10555G $>$ A are located in the transmembrane helical structure, while SNP m.10115G $>$ A is located in the outer surfaces of the inner mitochondrial membrane (Figure 3). The hydrophobicity of the COX-III protein was not changed by the Val62Ile amino acid change caused by variant $\mathrm{m} .10115 \mathrm{G}>\mathrm{A}$ (Figure 4), but this change at a key site of phospholipid binding may affect the combination of phospholipids related to adaptations to a hypoxic environment. Similarly, nonsynonymous substitutions Ser29Tyr (caused by

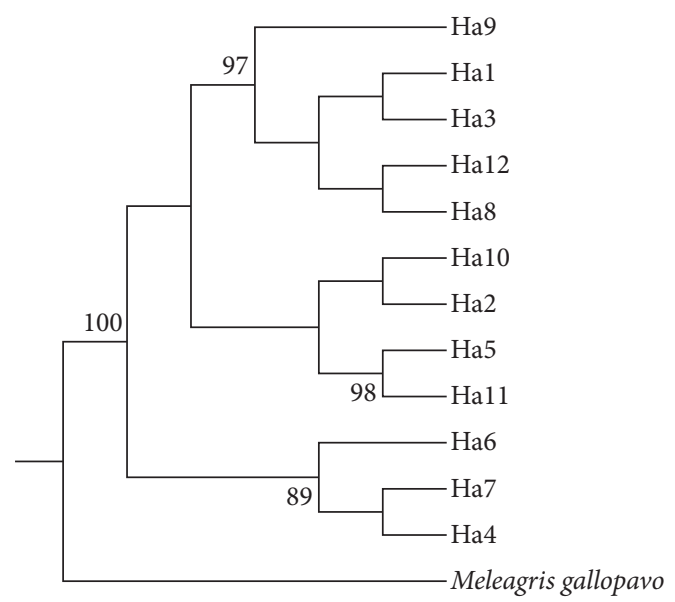

FIgURE 2: Constructing a phylogenetic tree using the Bayesian method. Note: phylogenetic tree of chicken based on haplotype sequence variation of COX-III gene; numbers at the nodes are bootstrap values.

SNP m. 10017C > A) and Ala162Thr (caused by m. 10555G >A) reduced the hydropathy index to some extent.

\section{Discussion}

Oxygen is one of the critical determinants for normal embryonic and foetal development. In avian embryos, a lack of oxygen causes high foetal mortality, heteroplasia, and cardiovascular dysfunction. The Tibetan chicken breed is native to Tibet and can survive with high hatchability regardless of the negative effects of hypoxia. Animals adapted to high altitudes are characterized by high haemoglobin concentrations and oxygen affinity [6], while lowland chicken breeds suffer polycythemia and ventricular hypertrophy at high altitudes [2, 4, 29]. 


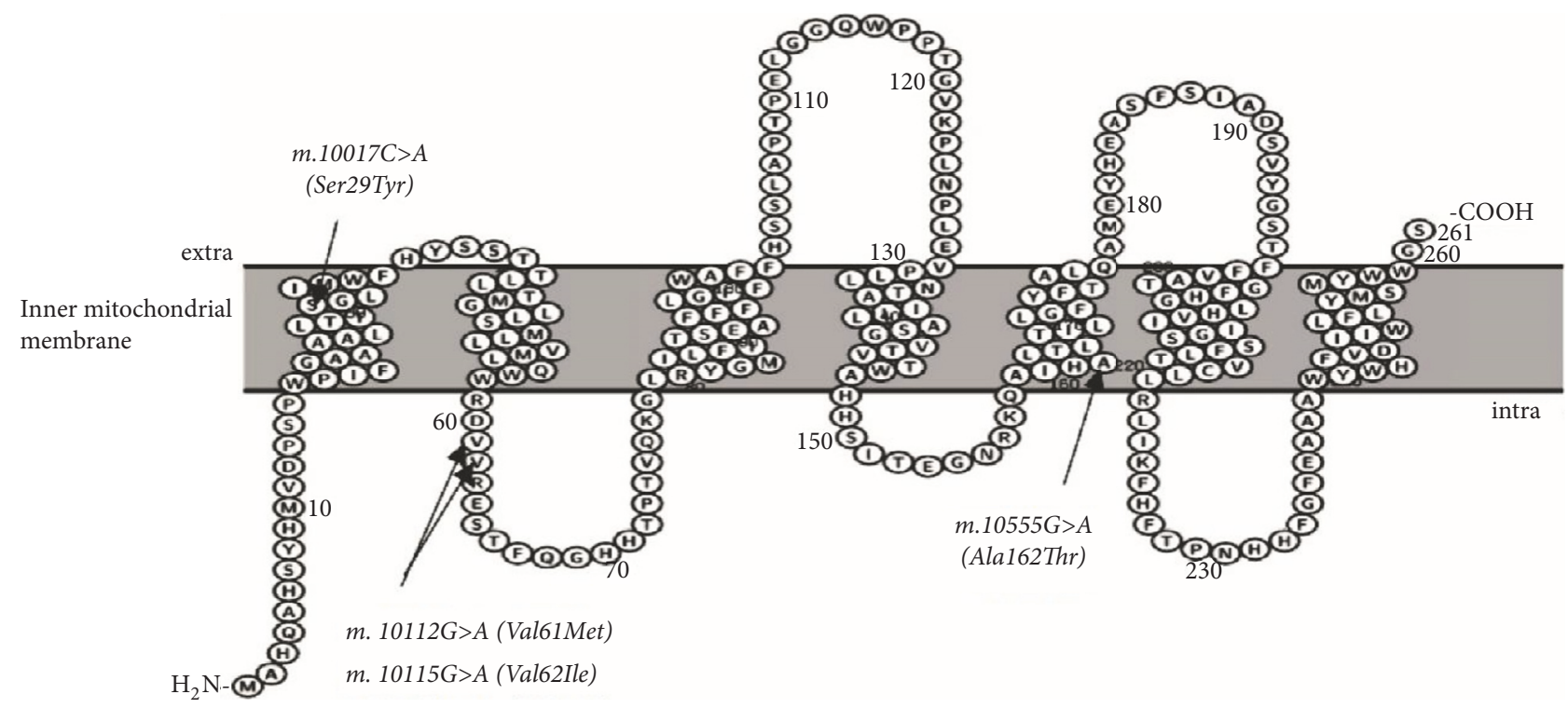

FIGURE 3: Diagram of transmembrane structure of COX-III protein predicted using the Protter program. The transmembrane structure of the coding sequence was the structure in Cochin-Chinese red jungle fowl. The transmembrane structure of the coding sequence was predicted by nonsynchronous mutations (m.10017C >A m.10112 G>A m.10115G > A m.10555G >A) in the present study.

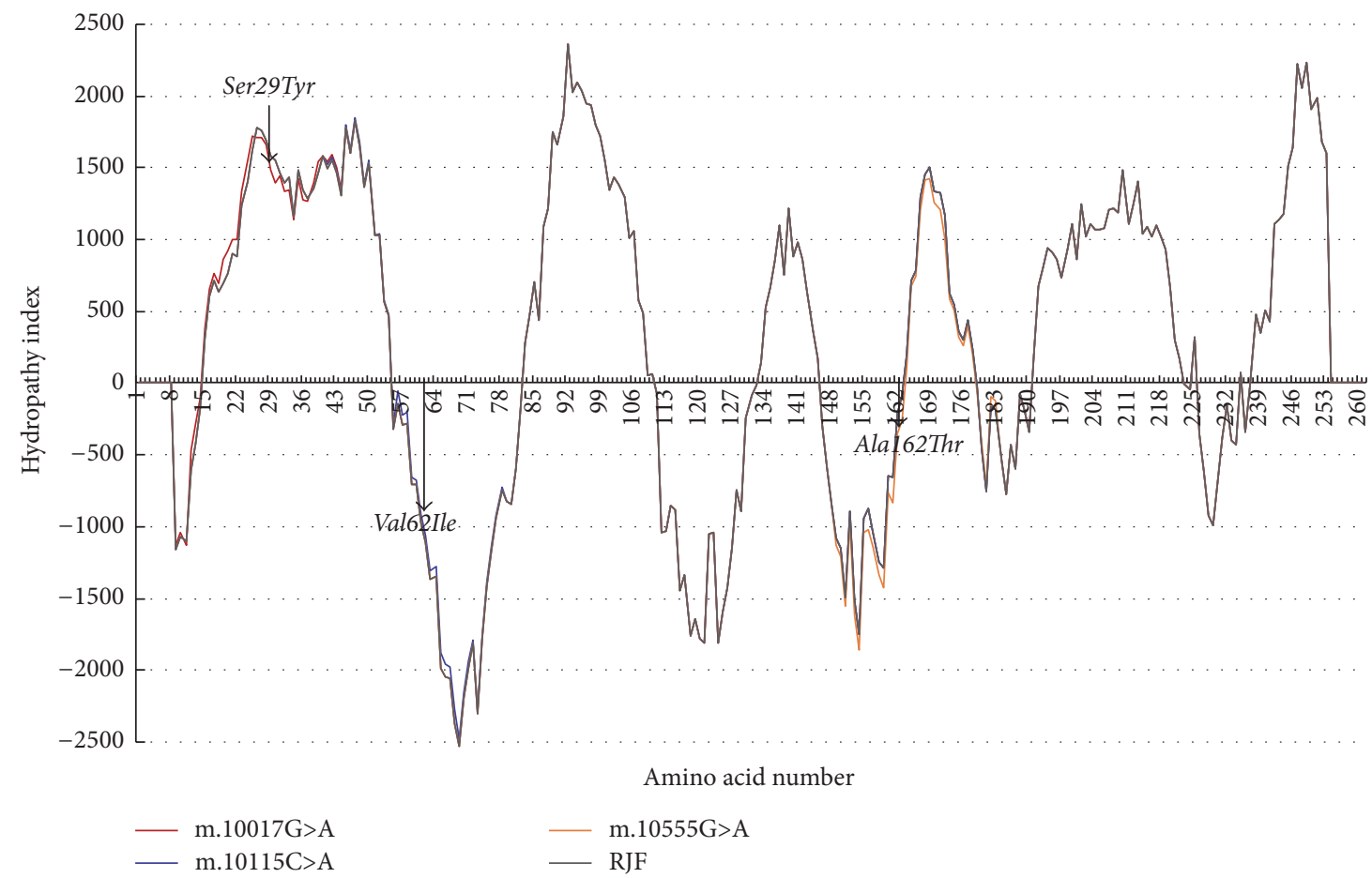

FIGURE 4: Negative values indicated interaction with water (hydrophilic), and positive values indicated getting out of water (hydrophobic). The red was the hydrophobic index of coding sequence with mutation $\mathrm{m} .10017 \mathrm{G}>\mathrm{A}$; the blue meant the hydrophobic index of coding sequence with mutation m.10115C $>\mathrm{A}$; the orange was the hydrophobic index of coding sequence with mutations m.10555G $>\mathrm{A}$; the irregular curve in gray was the hydrophobic index of coding sequence in the RJF.

By analyzing population haplotype diversity and the structure of chicken breeds in southwestern China based on COX-III sequences, we found that the Tibetan chicken has a higher level of haplotype diversity $(0.750 \pm 0.018)$ than lowland chickens $(0.570 \pm 0.028)$. For the results observed, we think that artificial selection leads to reduced nucleotide diversity in lowland chickens. Although 11 SNP sites were identified in COX-III from 12 geographic populations, the average genetic distance was only $0.196 \%$, revealing a low level of genomic polymorphisms in all populations. This low level 
of genetic diversity indicates that this gene is functionally important and hence has an evolutionary constraint. The 12 populations belong to neutral mutations $(P>0.1)$. This suggests that these populations are stable and have not undergone a population expansion in the past few years.

The analysis of the coefficient of differentiation and gene flow between populations suggests that some chicken populations have undergone genetic differentiation at places of equal altitude. This may reflect the introduction of varieties of different regions. For instance, the Tianfu white-bone fowl from a lower altitude has a close population genetic relationship with the Tibetan chicken (Gst $=0.01, N m=25.21$; $G s t=0.05, N m=4.9$ ), indicating that the Tianfu whitebone fowl was introduced from high altitude area. Moreover, Tibetan chickens from Haiyan and Doilungdêqên regions were most similar $(D a=0 \%, D x y=0.177 \%)$ suggesting that the genetic distance of the two regions is very close, and gene exchange is very rich (Gst $=0.01, N m=32.22)$.

The regulating mechanism in COX-III gene difference is still ambiguous between lowland and highland populations. A previous study detected SNPs in three mitochondrially encoded subunit genes of chicken $C O X$, including only one in COX-III (m.10081A $>\mathrm{G})$ between an expanded sample of 56 Tibetan chickens and 152 lowland birds [20]. Another study identified [19] eight SNPs, of which five (m.10081A $>$ G, m.10115G > A, m.10270G >A, m.10336A > G, and m.10447C>T) showed significant differences between Tibetan chickens and lowland chickens. Only the synonymous mutation m.10081A $>\mathrm{G}$ was found to differ between haplotypes H4 and $\mathrm{H} 5$, and chickens with the A allele at m.10081A $>\mathrm{G}$ had a probability of being over 2.6 times better adapted to hypoxia than those with the $G$ allele indicating that m.10081A $>$ G may be a prerequisite for shaping high altitude adaptation-specific haplotypes. Our focus on COX-III SNPs to explore the different haplotypes detected a novel mutation associated with high altitude adaptations. AMOVA showed that $C O X-I I I$ variation mainly existed within a population. Of the 12 defined haplotypes, the existence of $\mathrm{Ha} 4$, Ha7, Ha8, and HalO only in highland chickens and Ha9, Hall, and Ha12 only in lowland chickens indicates different degrees of genetic divergence between Tibetan chickens and lowland chickens. Ha1, Ha2, and $\mathrm{Ha} 4$ were found to be the earliest ancestors (Figure 1), while haplotype Hal was common to all populations suggesting that it is more stable and capable of adapting to new environmental selection. Moreover, Ha2 had significant relationship with high altitude adaptation $(P$ value, $1.911 \times 10^{-14^{*}}$; OR, 30.375, 95\% CI, 7.332-125.837), with the $\mathrm{C}$ allele at $\mathrm{m} .10587 \mathrm{~T}>\mathrm{C}$ was found to have a probability of being over 30.375 times better adapted to hypoxia than the $\mathrm{T}$ allele. We propose that $\mathrm{m} .10587 \mathrm{~T}>\mathrm{C}$ affects the functions of the COX enzyme in a similar way to the effect of m.10081A $>$ G [19] on high altitude adaptation of the Tibetan chicken. However, haplotype Hal simultaneously contained mutations m.10081A $>$ G and m.10587 $\mathrm{T}>\mathrm{C}$ and was negatively associated with high altitude adaptation. The function of these mutations warrants need to further research.

Three of the four nonsynonymous mutations identified in the present study (m.10017C $>$ A, m.10115G $>$ A, and
m.10555G >A) were peculiar to highland chickens. Mutation m.10115G $>$ A (with the allele frequency of $9.7 \%,{ }^{*} P<0.05$ ), shared by populations Ganzhi and Diqing, caused the amino acid mutation Val62Ile (Figure 1). A previous study reported SNP m.10115G $>$ A as an uncommon missense mutation in the Tibetan chicken mtDNA genome [30], while another study found that more than one-third of Tibetan chickens (44/125, 35.2\%) harboured this mutation, suggesting that it might be associated with high altitude adaptation [19]. In the current study, we found that mutation m.10115G $>A$ is a key site for phospholipid binding, suggesting that it impacts on the function of the mitochondrial membrane in Tibetan chicken. Whereas the other two nonsynonymous mutations (m.10017C > A m.10555G > A) and the reported SNP m.10065T $>C$ [19] both reduced hydropathy index to a certain extent.

\section{Conclusions}

In conclusion, we found largest genetic differentiation between Tibetan and lowland breeds and identified haplotype $\mathrm{Ha} 2$ is associated with Tibetan chicken populations. The possible association between increased hydrophobicity/reduced hydrophilic characteristics of the mitochondrial membrane and high altitude adaptation could provide a theoretical reference for poultry genetics. Therefore, our results provide a theoretical basis for future research into fowl breeding.

\section{Conflicts of Interest}

The authors declare that there are no conflicts of interest regarding the publication of this paper.

\section{Acknowledgments}

This work was supported by China Agricultural Research System (CARS-41), the 12th Five-Year Plan for Breeding Program in Sichuan-Selective Breeding of New Breeds and the Synthetic Strains in Laying Hens (2011NZ0099-7), National Natural Science Foundation of China (31402063), and Sichuan Provincial Department of Science and Technology Program (2015JQO023).

\section{References}

[1] Y.-W. Miao, M.-S. Peng, G.-S. Wu et al., "Chicken domestication: An updated perspective based on mitochondrial genomes," Heredity, vol. 110, no. 3, pp. 277-282, 2013.

[2] H. G. Bao, C. J. Zhao, J. Y. Li et al., "Sequencing and alignment of mitochondrial genomes of Tibetan chicken and two lowland chicken breeds," Science in China, Series C: Life Sciences, vol. 51, no. 1, pp. 47-51, 2008.

[3] X. Zhao, N. Wu, Q. Zhu, U. Gaur, T. Gu, and D. Li, "Highaltitude adaptation of Tibetan chicken from MT-COI and ATP6 perspective," Mitochondrial DNA, vol. 27, no. 5, pp. 3280-3288, 2016.

[4] H. Zhang, C. X. Wu, and Y. Z Chamba, "Adaptability to high altitude and NOS activity of lung in Tibetan chicken," Journal of China Agricultural University, vol. 11, no. 1, pp. 35-38, 2006. 
[5] M. Li and C. Zhao, "Study on Tibetan Chicken embryonic adaptability to chronic hypoxia by revealing differential gene expression in heart tissue," Science in China, Series C: Life Sciences, vol. 52, no. 3, pp. 284-295, 2009.

[6] C. Liu, L. F. Zhang, M. L. Song, H. G. Bao, C. J. Zhao, and N. Li, "Highly efficient dissociation of oxygen from hemoglobin in Tibetan chicken embryos compared with lowland chicken embryos incubated in hypoxia," Poultry Science, vol. 88, no. 12, pp. 2689-2694, 2009.

[7] Y. Liang, Z. Hua, X. Liang, Q. Xu, and G. Lu, "The crystal structure of bar-headed goose hemoglobin in deoxy form: The allosteric mechanism of a hemoglobin species with high oxygen affinity," Journal of Molecular Biology, vol. 313, no. 1, pp. 123-137, 2001.

[8] J. Zhang, Z. Hua, J. R. H. Tame, G. Lu, R. Zhang, and X. $\mathrm{Gu}$, "The crystal structure of a high oxygen affinity species of haemoglobin (bar-headed goose haemoglobin in the oxy form)," Journal of Molecular Biology, vol. 255, no. 3, pp. 484493, 1996

[9] Z.-Y. Zhang, B. Chen, D.-J. Zhao, and L. Kang, "Functional modulation of mitochondrial cytochrome c oxidase underlies adaptation to high-altitude hypoxia in a Tibetan migratory locust," Proceedings of the Royal Society B: Biological Sciences, vol. 280, no. 1756, Article ID 20122646, 2013.

[10] S. Kumar, "Patterns of nucleotide substitution in mitochondrial protein coding genes of vertebrates," Genetics, vol. 143, no. 1, pp. 537-548, 1996.

[11] P. Desjardins and R. Morais, "Sequence and gene organization of the chicken mitochondrial genome. A novel gene order in higher vertebrates," Journal of Molecular Biology, vol. 212, no. 4, pp. 599-634, 1990.

[12] E. Balsa, R. Marco, E. Perales-Clemente et al., "NDUFA4 is a subunit of complex IV of the mammalian electron transport chain," Cell Metabolism, vol. 16, no. 3, pp. 378-386, 2012.

[13] R. P. Kapur, "Emery and Rimoin's Principles and Practice of Medical Genetics, Fourth edition," Pediatric and Developmental Pathology, vol. 6, no. 2, pp. 204-205, 2003.

[14] M. BRUNORI, G. ANTONINI, F. MALATESTA, P. SARTI, and M. T. WILSON, "Cytochrome-c oxidase: Subunit structure and proton pumping," European Journal of Biochemistry, vol. 169, no. 1, pp. 1-8, 1987.

[15] G. R. Scott, P. M. Schulte, and S. Egginton, "Molecular evolution of cytochrome $\mathrm{C}$ oxidase underlies high-altitude adaptation in the bar-headed goose," Molecular Biology Evolution, vol. 28, no. 1, pp. 351-363, 2011.

[16] U.-R. Heinrich, J. Brieger, O. Selivanova et al., "COX-2 expression in the guinea pig cochlea is partly altered by moderate sound exposure," Neuroscience Letters, vol. 394, no. 2, pp. 121126, 2006.

[17] J. L. Park, J. A. Shayman, and L. Shu, "Differential involvement of COX1 and COX2 in the vasculopathy associated with the agalactosidase A-knockout mouse," American Journal of Physiology - Heart and Circulatory Physiology, vol. 296, no. 4, pp. H1133-H1140, 2009.

[18] S. A. Miller, D. D. Dykes, and H. F. Polesky, "A simple salting out procedure for extracting DNA from human nucleated cells," Nucleic Acids Research, vol. 16, no. 3, article 1215, 1988.

[19] J. Sun, H. Zhong, S.-Y. Chen, Y.-G. Yao, and Y.-P. Liu, "Association between MT-CO3 haplotypes and high-altitude adaptation in Tibetan chicken," Gene, vol. 529, no. 1, pp. 131-137, 2013.

[20] H. Bao, C. Zhao, L. Zhang, J. Li, and C. Wu, "Single-nucleotide polymorphisms of mitochondrially coded subunit genes of cytochrome c oxidase in five chicken breeds," Mitochondrial DNA, vol. 19, no. 5, pp. 461-464, 2008.

[21] K. Tamura, D. Peterson, N. Peterson, G. Stecher, M. Nei, and S. Kumar, "MEGA5: molecular evolutionary genetics analysis using maximum likelihood, evolutionary distance, and maximum parsimony methods," Molecular Biology and Evolution, vol. 28, no. 10, pp. 2731-2739, 2011.

[22] P. Librado and J. Rozas, "DnaSP v5: a software for comprehensive analysis of DNA polymorphism data," Bioinformatics, vol. 25, no. 11, pp. 1451-1452, 2009.

[23] L. Excoffier, G. Laval, and S. Schneider, "Arlequin (version 3.0): an integrated software package for population genetics data analysis," Evolutionary Bioinformatics Online, vol. 1, no. 4A, pp. L418-L421, 2005.

[24] J. W. Streicher, A. J. Crawford, and C. W. Edwards, "Multilocus molecular phylogenetic analysis of the montane Craugastor podiciferus species complex (Anura: Craugastoridae) in Isthmian Central America," Molecular Phylogenetics and Evolution, vol. 53, no. 3, pp. 620-630, 2009.

[25] D. Posada and K. A. Crandall, "MODELTEST: testing the model of DNA substitution," Bioinformatics, vol. 14, no. 9, pp. 817-818, 1998.

[26] L. Fan and Y.-G. Yao, "MitoTool: A web server for the analysis and retrieval of human mitochondrial DNA sequence variations," Mitochondrion, vol. 11, no. 2, pp. 351-356, 2011.

[27] U. Omasits, C. H. Ahrens, S. Müller, and B. Wollscheid, "Protter: Interactive protein feature visualization and integration with experimental proteomic data," Bioinformatics, vol. 30, no. 6, pp. 884-886, 2014.

[28] K. Hofmann, "TMBASE-A database of membrane spanning protein segments," Biol. Chem. Hoppe-Seyler, vol. 374, no. 1, p. $1,1993$.

[29] C. Monge, F. Le, and F. León-Velarde, "Le $\leq$ n-Velarde, Physiological adaptation to high altitude: Oxygen transport in mamals and birds," Physiological Reviews, vol. 71, no. 4, pp. 1135-1172, 1991.

[30] X. M. Tong, Y. Liang, and W. Wang, "[Complete sequence and gene organization of the Tibetan chicken mitochondrial genome]," Hereditas, vol. 28, no. 7, pp. 769-777, 2006. 

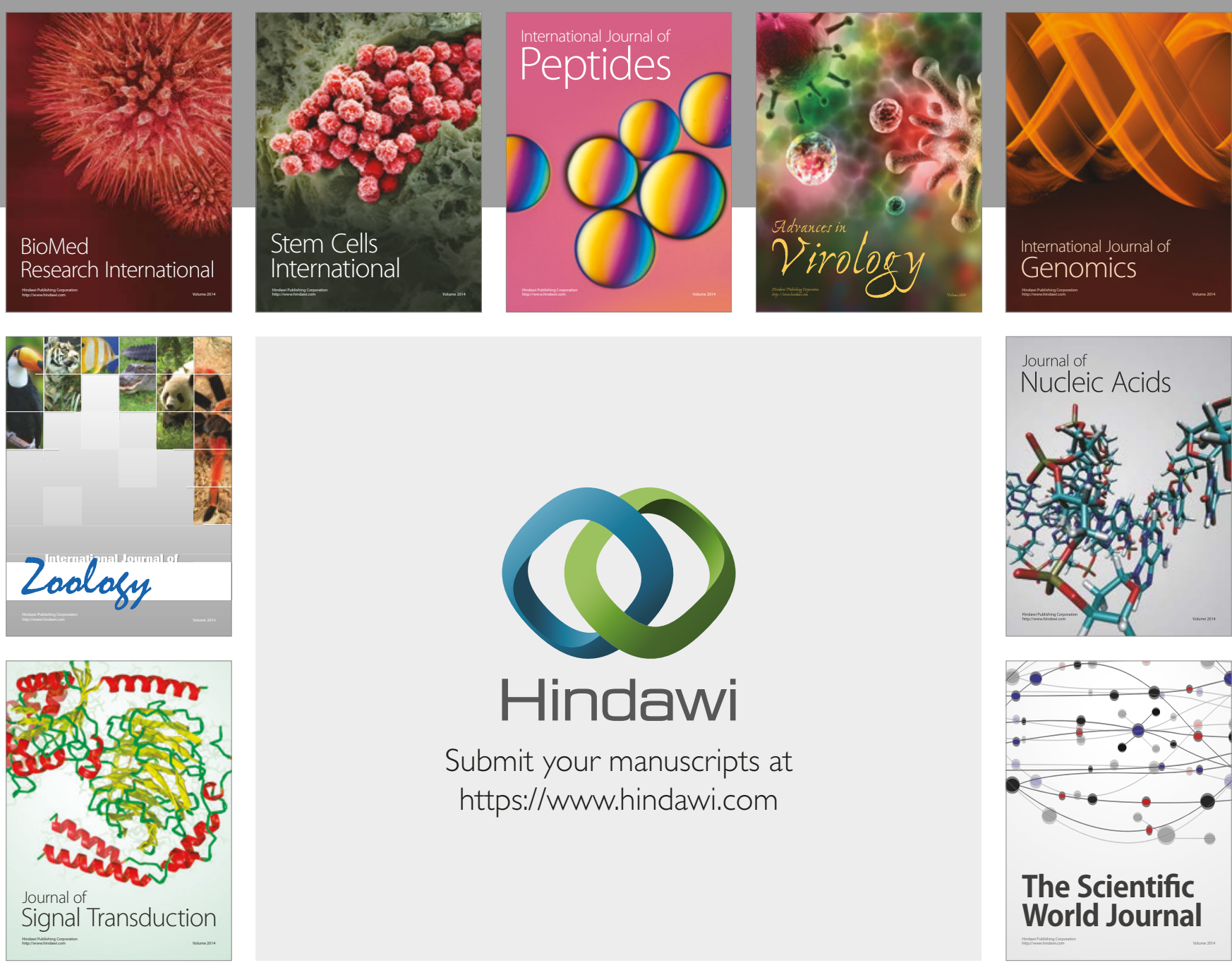

Submit your manuscripts at

https://www.hindawi.com
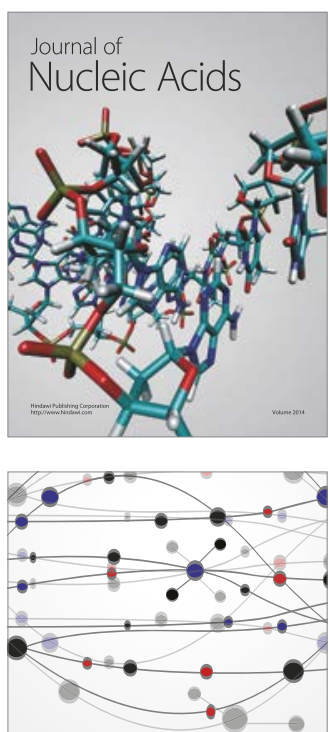

The Scientific World Journal

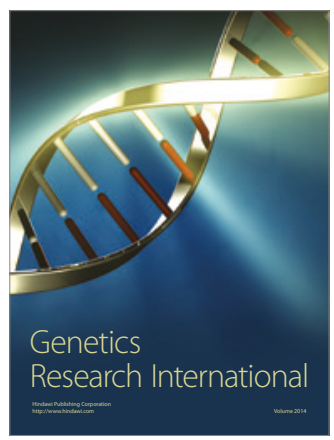

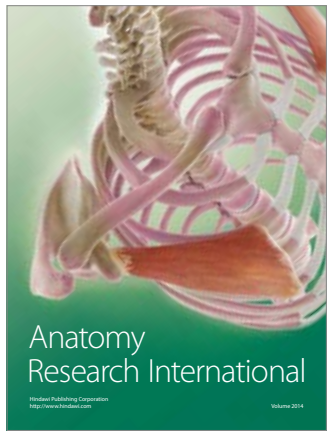

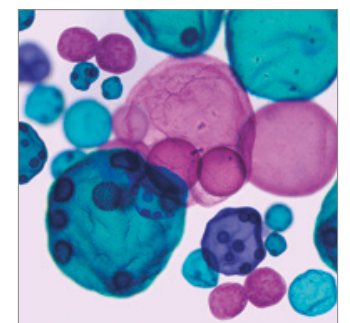

International Journal of Microbiology
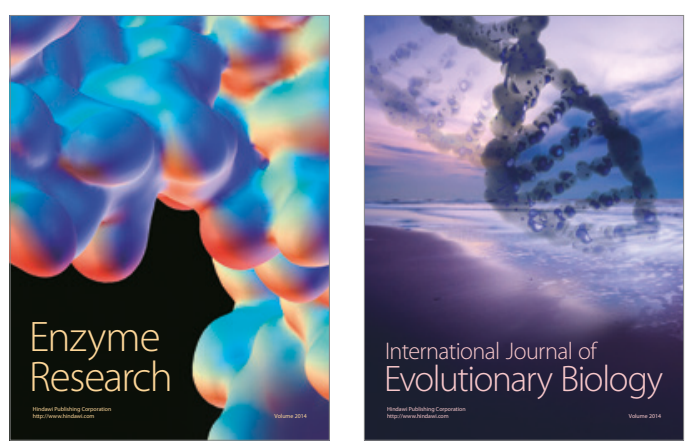
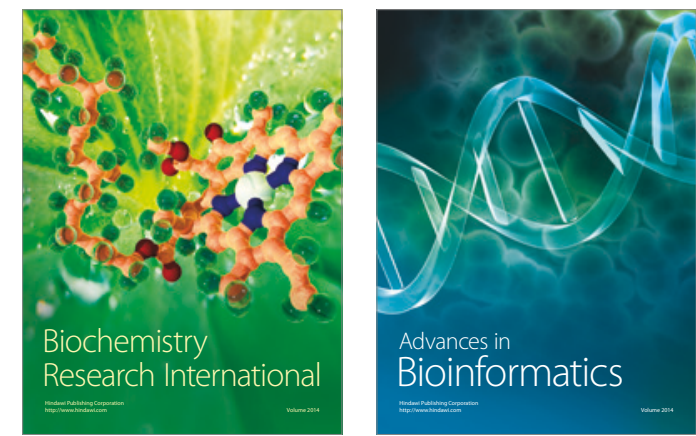

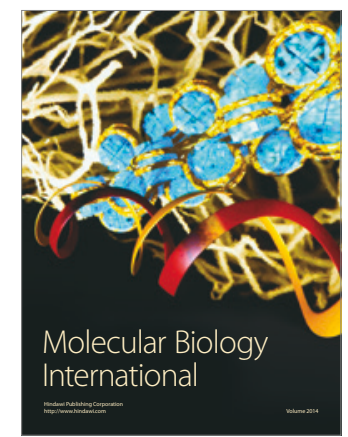

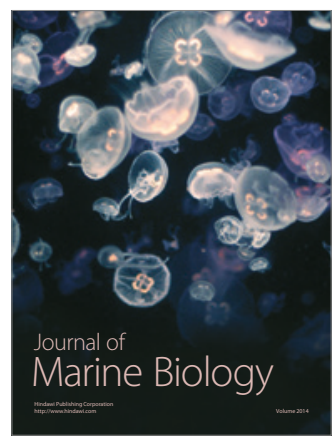

